\title{
Identification of a novel transcript isoform of the TTLL12 gene in human cancers
}

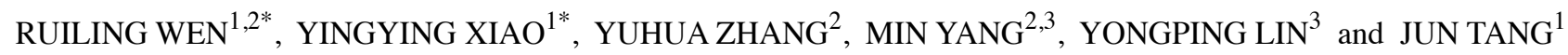 \\ ${ }^{1}$ KingMed Diagnostics and KingMed School of Laboratory Medicine, Guangzhou Medical University, Guangzhou, \\ Guangdong 510330; ${ }^{2}$ Cytate Institute for Precision Medicine and Innovation, Guangzhou Cytate Biomedical \\ Technologies Inc., Guangzhou, Guangdong 510663; ${ }^{3}$ Department of Clinical Laboratory and Research Center \\ of Translational Medicine, The First Affiliated Hospital of Guangzhou Medical University, \\ Guangzhou, Guangdong 510120, P.R. China
}

Received June 16, 2016; Accepted July 28, 2016

DOI: $10.3892 /$ or.2016.5135

\begin{abstract}
Tubulin tyrosine ligase like 12 (TTLL12), a member of the tubulin tyrosine ligase (TTLL) family, has not been completely characterized to date. It is reported that histone methylation, tubulin modifications, mitotic duration and chromosome ploidy play crucial roles in a variety of cancers, and are related to tumorigenesis and cancer progression. A recent study showed that TTLL12 may be a pseudo-enzyme which has a SET-like domain and a TTL-like domain. In the present study, we first used 3'-rapid amplification of cDNA ends (3'-RACE) to amplify the transcripts of the TTLL12 gene from a human lung cancer cell line H1299, and unexpectedly discovered a new transcript isoform characterized with an additional 108-bp nucleotide sequence inserted at the location from 902 to 903 bases of the TTLL12 coding sequence (CDS), where it also locates between exons 5 and 6. Next, utilizing RT-PCR and Sanger sequencing, we further confirmed the existence of such a new transcript isoform of TTLL12 in more human cancer cells including lung cancer cells and other cancer cells. Moreover, several lung cancer cell lines were found to display a much higher proportion of the new isoform compared with TTLL12 wild-type transcript. These results suggest that the new TTLL12 isoform may be of importance for proper maintenance of lung cancer cells. Therefore, the new isoform of TTLL12, with the inserted sequences probably acting as a disordered region, provides a novel perspective regarding TTLL12 functions in human cancers including lung cancer.
\end{abstract}

Correspondence to: Professor Jun Tang, KingMed Diagnostics and KingMed School of Laboratory Medicine, Guangzhou Medical University, 2429 XinGang Dong Road, Guangzhou, Guangdong 510330, P.R. China

E-mail: jtanguc@gmail.com

${ }^{*}$ Contributed equally

Key words: TTLL12, 3'-RACE, alternative splicing, disordered region, cancers

\section{Introduction}

Tubulin tyrosine ligase like 12 (TTLL12) is the least characterized and the most divergent member of the TTLL family, which possesses catalytic functions in tubulin post-translational modifications (1-3). However, TTLL12 is said to be a pseudoenzyme having a phylogenetically conserved association of two nonfunctional domains including a SET-like domain and TTL-like domain, for which several differences exist in the structure, compared with other members of the TTLL family (4). These two domains are associated with histone methylation and tubulin modification, respectively. Recent studies have shown that TTLL12 could affect histone methylation, tubulin modification, mitotic duration and chromosome ploidy in human larynx cancer cells (5-8). In addition, there are numerous studies that have revealed that the TTLL family is closely linked to human cancer, such as neuroblastomas (9-11). For example, the TTLL family is often suppressed in human cancer (10) and is positively correlated with a poor prognosis in breast cancer (11). In prostate cancer, the level of TTLL12 expression was found to be increased in the proliferating layer of benign tissue and during cancer progression to metastasis $(12,13)$. These findings suggest that TTLL12 may display the same pattern in other types of cancers, and it could play a crucial role in tumorigenesis and tumor progression.

In the present study, we found a new transcript isoform of the TTLL12 gene, namely the 36 amino acid (AA) plus isoform, with an additional 108-bp nucleotide sequence inserted between exons 5 and 6 of the wild-type. This new isoform may be the result of alternative splicing, and we identified it in several lung cancer cell lines as well as other cancer cell lines. Notably, some of the lung cancer cell lines presented a much higher proportion of the 36AA plus isoform compared with the wild-type TTLL12 transcript, implying the potential role of the new isoform in lung cancer cells. Moreover, the inserted 36AA was predicted to be part of a disordered region, which can alter the molecular structure of the whole protein, and could affect adaptive and deleterious-on gene expression and function.

Collectively, our discovery of a new transcript isoform of TTLL12 with an additional $108 \mathrm{bp}$, predicted to be a disordered 
region, provides an alternative prospective to study the functions of TTLL12 in tumor development and progression. In addition, our findings may also open a new window to explore potential targets related to post-translational modification of tubulin, which may eventually contribute to the development of more selective agents for cancer therapy.

\section{Materials and methods}

Cell culture. Human lung cancer cell lines (H1299, 95-D, SPCA-1, A549, SK-MES-1, PC-9, H2170 and Hcc-827), human esophageal cancer cell lines (TE-11 and EC-109), human normal esophageal cell lines (HET-1A and HEEpiC), human acute monocytic leukemia cell line (THP-1), and human breast cancer cell line (MCF-7) were purchased from the Cell Bank of the China Academy of Science (Shanghai, China). THP-1, EC-109 and all the human lung cancer cell lines were grown in RPMI-1640 medium (Life Technologies, Carlsbad, CA, USA) supplemented with $10 \%$ fetal bovine serum (FBS; Gemini, Woodland, CA, USA), $100 \mathrm{IU} / \mathrm{ml}$ penicillin and $100 \mu \mathrm{g} / \mathrm{ml}$ streptomycin. TE-11 and MCF-7 cells were grown in Dulbecco's modified Eagle's medium (DMEM) culture medium (Life Technologies) supplemented with $10 \%$ FBS, $100 \mathrm{IU} / \mathrm{ml}$ penicillin and $100 \mu \mathrm{g} / \mathrm{ml}$ streptomycin. HET-1A and HEEpiC were grown in EpiCM-2 complete medium (ScienCell, Carlsbad, CA, USA) with $100 \mathrm{IU} / \mathrm{ml}$ penicillin and $100 \mu \mathrm{g} / \mathrm{ml}$ streptomycin. All of the cells were cultured at $37^{\circ} \mathrm{C}$ in a humidified incubator with $5 \% \mathrm{CO}_{2}$.

RNA isolation. Total RNA was isolated from the cell cultures using TRIzol (Life Technologies) according to the manufacturer's instructions. The total RNA was quantified based on absorbance at $260 \mathrm{~nm}$ and the integrity of purified RNA was confirmed by agarose gel electrophoresis with a $28 \mathrm{~S} / 18 \mathrm{~S}$ ratio not $<1$. The total RNA was stored at $-80^{\circ} \mathrm{C}$.

3'-Rapid amplification of cDNA ends (3'-RACE). As displayed in Fig. 1, the full length of TTLL12 cDNA was amplified by 3'-RACE (SMARTer RACE 3' kit; Takara, Dalian, China). First-strand cDNA synthesis from total RNA of H1299 was performed using a traditional reverse transcription procedure, but with a special oligo(dT) primer: [3'-RACE CDS primer, 5'-AAGCAGTGGTATCAACGCAGAGTAC (T) 30VN-3'; $\mathrm{N}=\mathrm{A}, \mathrm{C}, \mathrm{G}$ or $\mathrm{T} ; \mathrm{V}=\mathrm{A}, \mathrm{G}$, or C]. The first-strand cDNA synthesis reaction products were diluted with $10 \mu \mathrm{l}$ Tricine-EDTA Buffer (Takara). The diluted first-strand cDNA was used as template and second-strand synthesis was amplified with a 3 gene-specific primer (3' GSP, 5'-GATTACGCCAAGCTTAG AGCACACAGACGGCGCGGGTG-3') and universal primer mix A (UPM; long, 5'-CTAATACGACTCACTATAGGGCA AGCAGTGGTATCAACGCAGAGT-3' and short, 5'-CTAAT ACGACTCACTATAGGGC-3'). The 3'-RACE DNA samples were electrophoresed on an agarose gel. The position of the desired fragment was located under UV light and the products were extracted with the NuceloSpin Gel and PCR Clean-Up kit (Takara).

In-fusion cloning of RACE products. The 3'-RACE products were cloned into a pUC19 vector by the In-Fusion HD Cloning kit (Takara) according to the protocol. The recombinant vectors were then transformed into Stellar Competent Cells (Takara) and the bacteria was spread on LB plates containing $100 \mu \mathrm{g} /$ $\mathrm{ml}$ of ampicillin. All of the plates were incubated overnight at $37^{\circ} \mathrm{C}$ and individual isolated colonies were randomly selected from each experimental plate. Plasmid DNA was isolated using HiPure Plasmid EF Micro kit (Magen, Suzhou, China) according to the manufacturer's instructions.

$R T-P C R$. First-strand cDNA from total RNA was constructed by Transcript II All-in-One First-Strand cDNA Synthesis SuperMix kit (Life Technologies) following the manufacturer's instructions. The first-strand cDNA was then used as a template for PCR and the specific primers used were as follows: TTLL12 sense primer 1, 5'-GAAGATGCCGGTGTGGTATA-3' and TTLL12 antisense primer 1, 5'-CACGTCCGTGTAGACCT TGA-3'; TTLL12 sense primer 2, 5'-GAGACTTTGCCTACG GAGAGA-3' and TTLL12 antisense primer 2, 5'-GCTGAG TTTCCTGTAGTCCTTGA-3'. Meanwhile, we used pUC19 vectors inserted with TTLL12 wild-type or TTLL12 36AA plus isoform as positive templates for PCR amplification of TTLL12 wild-type or 36AA plus isoform, respectively. PCR products were characterized by electrophoresing on agarose gel. The desired position was determined under UV light and PCR products were isolated by NuceloSpin Gel and PCR Clean-Up kit.

Cloning and sequencing. PCR products were cloned into a pMD18-T Simple Vector (Takara) and transformed into chemically competent cells. Individual colonies were grown overnight at $37^{\circ} \mathrm{C}$ and plasmid DNA was isolated using HiPure Plasmid EF Micro kit according to the protocol, and processed to Sanger sequencing (Life Technologies).

Bioinformatics analysis.Protein structures of the TTLL12 wildtype and new TTLL12 36AA plus isoform were predicted by bioinformatics softwares. The mRNA CDS and AA sequence of wild-type TTLL12 were gained in NCBI (http://www.ncbi. nlm.nih.gov/blast). In addition, the new transcript of TTLL12 was translated into a new AA sequence using DNAStar EditSeq. Then, the AA sequence of the new TTLL12 36AA plus isoform was aligned with the wild-type by NCBI protein blast (http://blast.ncbi.nlm.nih.gov/). Physicochemical properties of the wild-type and new isoform were predicted by online tools ProtParam and ProtScale (http://www.expasy.ch/tools/ protscale.html), respectively. Secondary structures of the wild-type and new isoform AA sequences were predicted by DNAStar Protean and online tool CFSSP (http://www.biogem. org/tool/chou-fasman/). To further investigate the function of the new isoform of the TTLL12 transcript, we also used the online prediction tool DisPort search (http://www.disport.org/ search.php) to predict whether the additional 36 AA sequence is in a disordered region of TTLL12.

\section{Results}

The full length of TTLL12 cDNA amplified by 3'-RACE from total RNA of H1299 cells. To gain the full length cDNA of TTLL12, we first generated the first-strand cDNA from total RNA of H1299 cells, and then the first-strand cDNA was used as the template to amplify the second-strand cDNA using 


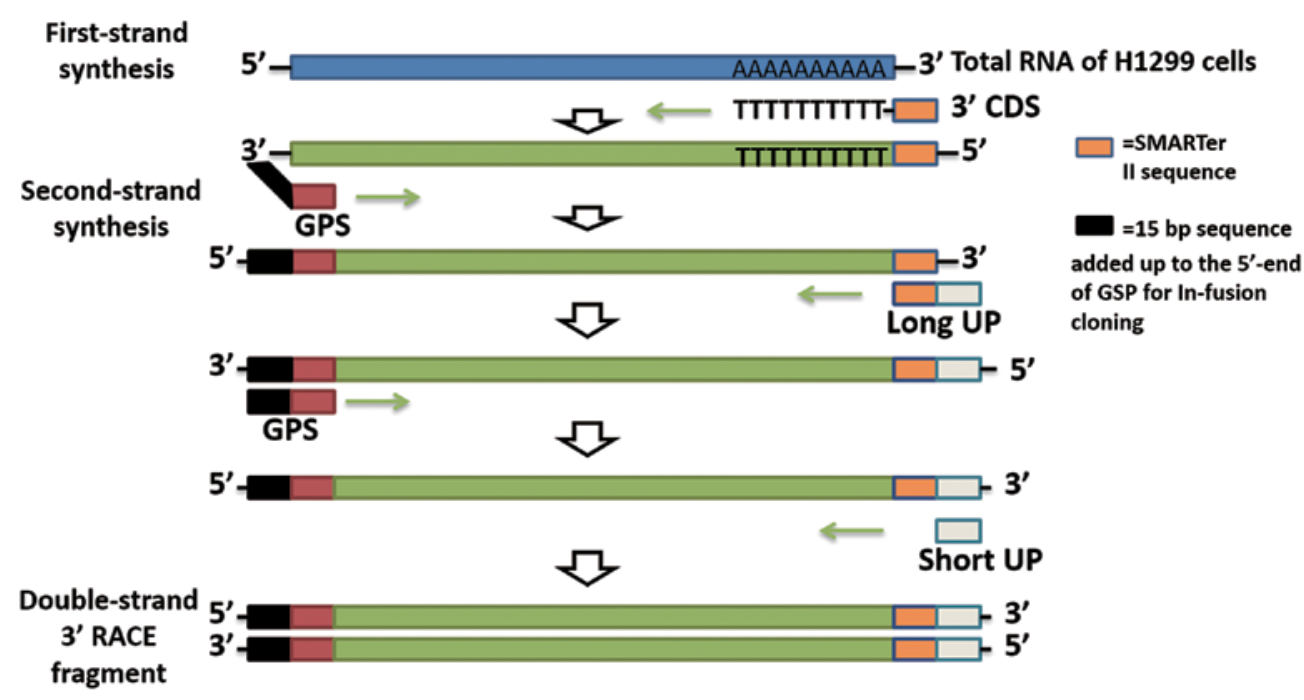

Figure 1. The procedure of the 3'-rapid amplification of cDNA ends. The 3'-RACE first-strand cDNA was synthesized using a traditional reverse transcription procedure, but with a special oligo(dT) primer ( $\left.3^{\prime} \mathrm{CDS}\right)$. The first-strand cDNA was used as the template for the second-strand synthesis. The second-strand cDNA was generated with a distinct gene-specific primer (GSP) and universal primers mix A (UPM, containing long universal primer and short universal primer). The GSP and UPM ensure the full length of TTLL12 cDNA can be synthesized.

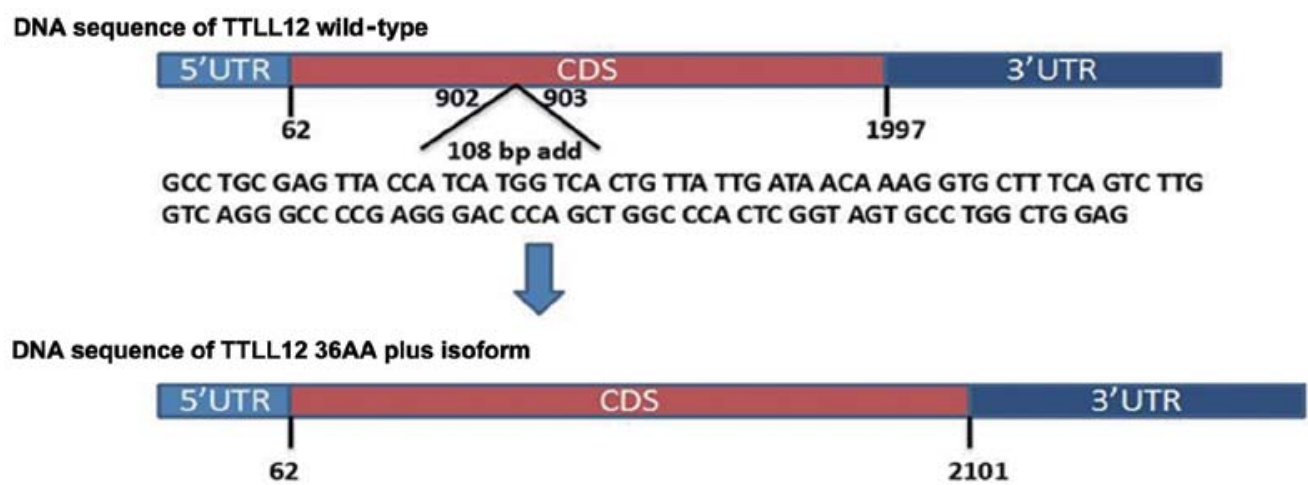

Figure 2. Diagrams for DNA sequences of the TTLL12 wild-type and the new TTLL12 36AA plus isoform. The new TTLL12 36AA plus isoform was found to contain an additional 108-bp nucleotide sequence which was located in the CDS from nucleotides 902 to 903 , between exons 5 and 6 , when aligned with the GenBank consensus Homo sapiens TTLL12 genomic sequence.

3'-RACE (Fig. 1). We aligned the 3'-RACE sequences with the consensus Homo sapiens TTLL12 genomic sequence (wildtype) from GenBank, and then found an additional 108-bp nucleotide sequence located at CDS from 902 to 903 bases, between exons 5 and 6 (Fig. 2). This new isoform was called the 36AA plus isoform of TTLL12.

Detection of the new TTLL12 36AA plus isoform in both lung cancer cell lines and other cancer cell lines. To determine whether the TTLL12 36AA plus isoform exists in human cancers, we isolated total RNAs from 8 human lung cancer cell lines (H1299, 95-D, SPCA-1, A549, SK-MES-1, PC-9, H2170 and Hcc-827), 2 human esophageal cancer cell lines (TE-11 and EC-109), 2 human normal esophageal cell lines (HET1A, HEEpiC), human acute monocytic leukemia cell line THP-1 and human breast cancer cell line MCF-7. The reverse transcriptase-PCR products were then used as the template for PCR. In PCR, the plasmids harboring the 36AA plus isoform and wild-type of the TTLL12 gene sequence were used as the positive templates for the 36AA plus isoform and wild-type, respectively. All the PCR products were electrophoresed on agarose gel. We then located the position of the desired fragment under UV light and extracted the PCR products from the gel. Extracted PCR products were subsequently cloned into a pMD18-T Simple Vector and transformed into chemically competent cells. All the plasmids from the positive clone were sequenced and aligned with the TTLL12 36AA plus isoform and TTLL12 wild-type. As a result, we found the product of the TTLL12 36AA plus isoform in all of the detected cell lines, and 3 lung cancer cell lines (H1299, H2170 and Hcc-827) showed the highest proportion of the 36AA plus isoform compared to the wild-type (Fig. 3). In addition, the sequencing results of the plasmids extracted from the positive clone were aligned, and demonstrated that the PCR products were from the TTLL12 36AA plus isoform (Fig. 4). Significantly, the TTLL12 36AA plus isoform was expressed more abundantly than the wildtype in the human cancer cell lines, particularly in the human lung cancer cell lines, as compared with the human normal 
A
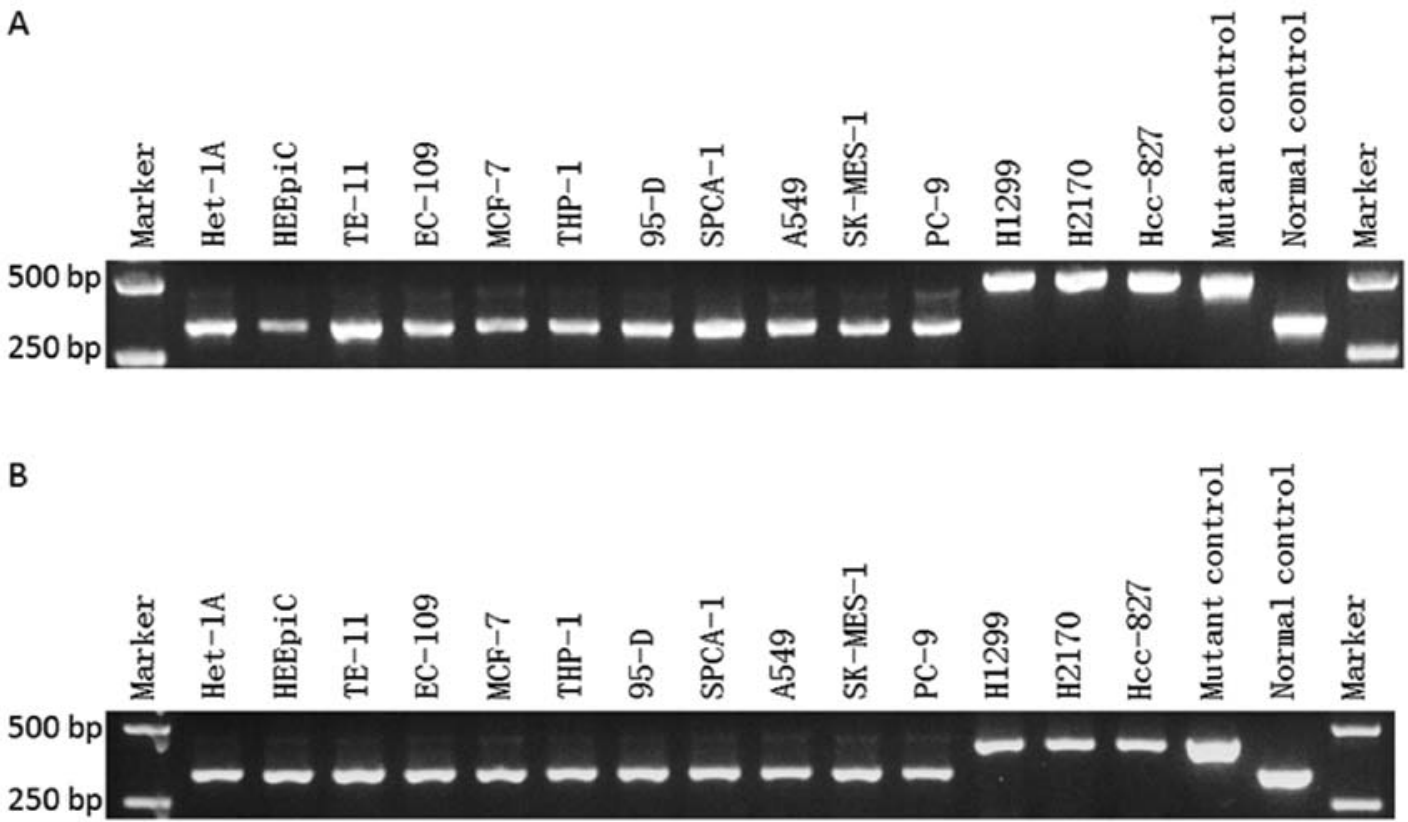

Figure 3. Detection of the new TTLL12 36AA plus isoform in human cancer cell lines. RT-PCR was employed in a series of cell lines including human lung cancer cell lines (H1299, 95-D, SPCA-1, A549, SK-MES-1, PC-9, H2170 and Hcc-827), human esophageal cancer cell lines (TE-11 and EC-109), human normal esophageal cell lines (HET-1A and HEEpiC), human acute monocytic leukemia cell line THP-1, and human breast cancer cell line MCF-7. PCR was performed using (A) TTLL12 sense primer 1 and TTLL12 antisense primer 1, and (B) TTLL12 sense primer 2 and TTLL12 antisense primer 2. The PCR product for positive control of the 36AA plus isoform was $484 \mathrm{bp}$ in size while the size of the wild-type product was $376 \mathrm{bp}$, when the pair of primer 1 was used. The product for positive control of the 36AA plus isoform was $434 \mathrm{bp}$ in size while the wild-type was $326 \mathrm{bp}$ in size as the pair of primer 2 was used.

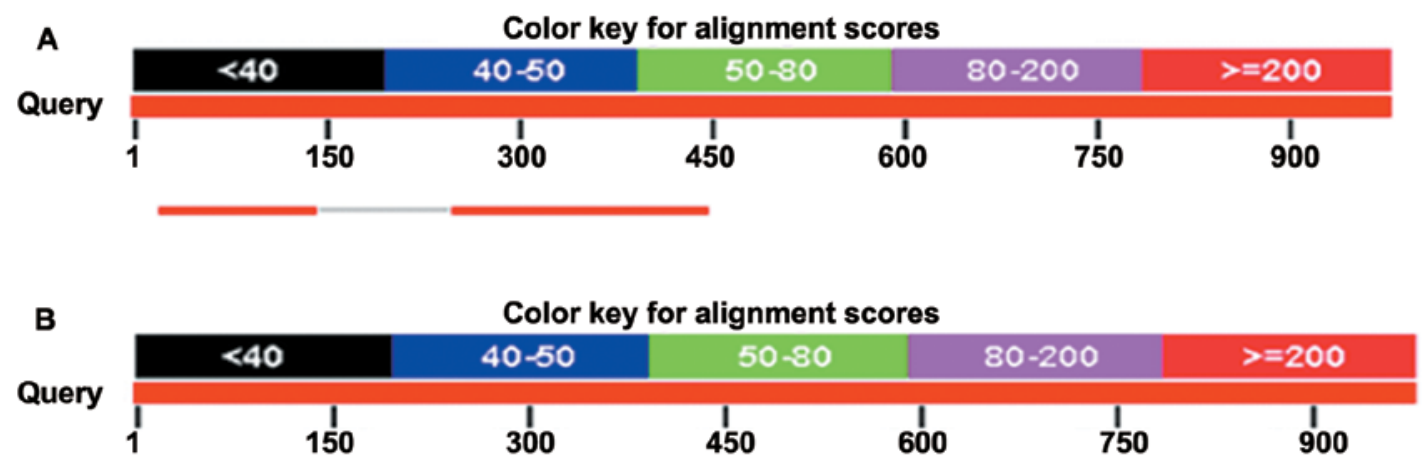

Figure 4. Alignment of DNA sequences of the TTLL12 PCR products. DNA sequences of the TTLL12 PCR products were aligned between (A) TTLL12 wild-type and (B) new TTLL12 36AA plus isoform.

esophageal cell lines. The findings indicate that the new TTLL12 36AA plus isoform may play an important role in the development of various tumors.

Physicochemical properties of the TTLL12 wild-type and new 36AA plus isoform. The AA sequence of the TTLL12 36AA plus isoform was obtained using DNAStar EditSeq and translating the DNA sequences. Then, the AA sequence of the new isoform was aligned with the TTLL12 wild-type, and we found an additional 36AA inserted at the location of 281AA of the TTLL12 wild-type (Fig. 5A). Next, we predicted the physicochemical properties by ProtParam and ProtScale. The properties of TTLL12 wild-type and 36AA plus isoform AA sequences were predicted (Table I) as follows: the total number of negatively charged residues $(\mathrm{Asp}+\mathrm{Glu})$ was
93 and 96; and grand average of hydropathicity (GRAVY) was -0.390 and -0.338 . In addition, the total hydropathicities of the two types of TTLL12 proteins are displayed in diagram (Fig. 5B and C). These results suggest that the two types of TTLL12 proteins are hydropathical molecules, and the 36AA plus isoform is more stable than the wild-type.

Secondary structures of TTLL12 wild-type and new 36AA plus isoform. Secondary structures of the TTLL12 wild-type and TTLL12 36AA plus isoform were predicted by DNAStar Protean and online tool CFSSP. As a result, the total residues of $\alpha$-Helix , $\beta$-sheet and $\beta$-turn in the TTLL12 wild-type were 476, 428 and 72, and the corresponding percentages were 73.9, 66.5 and $11.2 \%$, respectively (Fig. 6A). While total residues of $\alpha$-Helix, $\beta$-sheet and $\beta$-turn in the TTLL12 36AA plus isoform 
A

TTLL12 36AA plus isoform

TTLL12 wild-type
241
DFAYGETDPLIRKCMLLPWAPTDMLDLSSCTPEPPAEHTQACELPSWSLLLITKVLSVLV DFAYGETDPLIRKCMLLPWAPTDMLDLSSCTPEPPAEHTQA

TTLL12 36AA plus isoform TTLL12 wild-type

300

281

01 RAPRDPAGPLGSAWLEAILEENKEKLPLDINPWHPHGHIFKVTTDVQVASSLTHPRFT 360 ILEENKEKLPLDINPWHPHGHIFKVYTDVQQVASSLTHPRFT

282 -ILEENKEKLPLDINPVVHPHGHIFKVYTDVQQVASSLTHPRFT 324

\section{B}

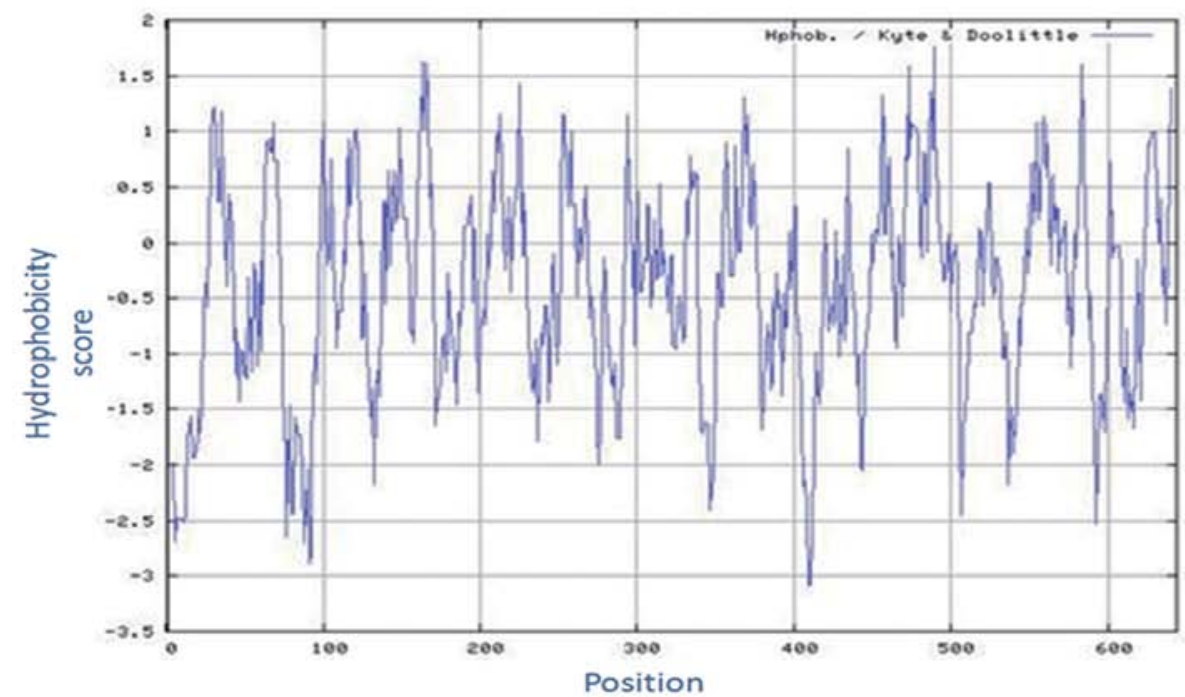

C

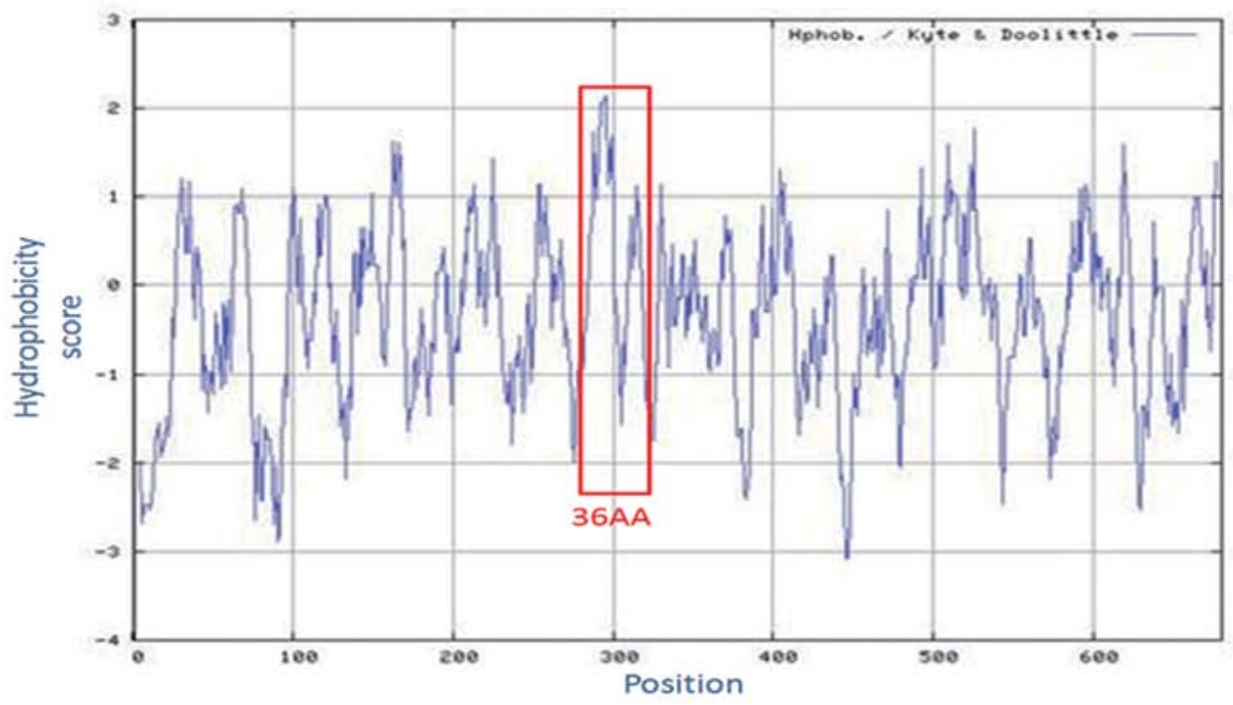

Figure 5. Analysis of the amino acid sequences of the TTLL12 wild-type and new TTLL12 36AA plus isoform. The amino acid sequence of the new TTLL12 36AA plus isoform was aligned with the TTLL12 wild-type by NCBI protein blast, and an additional 36 amino acid sequence was found (A). Hydropathicity of the wild-type (B) and the new 36AA plus isoform (C, red box indicates the inserted 36AA peptide) of TTLL12 was predicted by ProtParam and ProtScale, respectively.

were 504,455 and 75 , and the corresponding percentages were 74.1,66.9 and 11.0\%, respectively (Fig. 6B). The results showed that the proportions of different types of secondary structures in these two TTLL12 proteins were slightly different (Table II).

The additional 36AA is involved in a disordered region of TTLL12. To ascertain whether the additional 36AA in the
TTLL12 36AA plus isoform is in a disordered region of TTLL12, we firstly sought the disordered regions of the TTLL12 36AA plus isoform by the opening accessible online resources DisProt search, and found that the peptide of 260AA to 299AA in the TTLL12 36AA plus isoform was highly identical to the disordered region of Bcl-2-like protein 11 (B2L11) (Fig. 7A), suggesting this peptide could act as a disordered region in the 

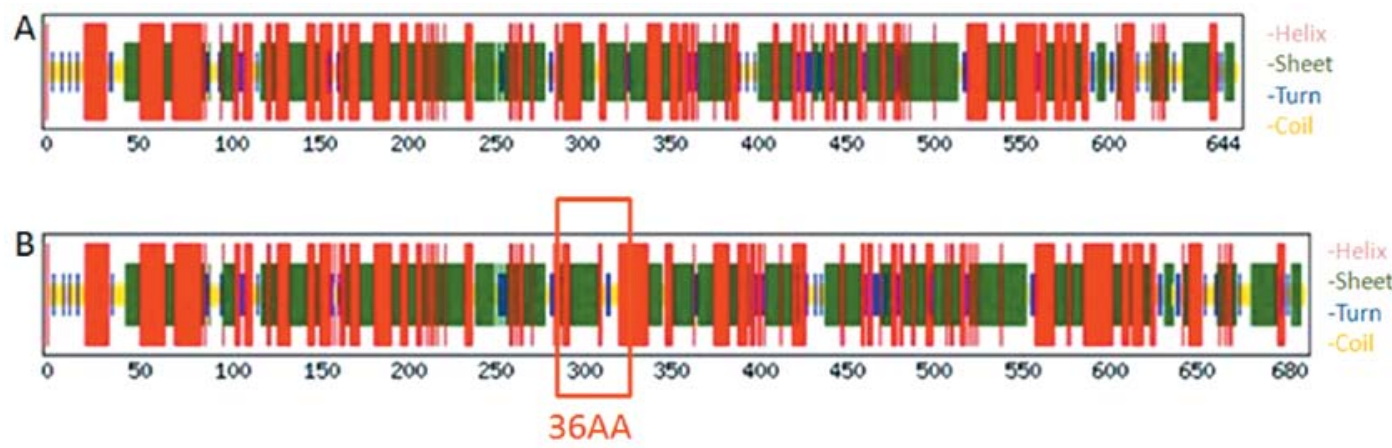

Figure 6. Secondary structures of the TTLL12 wild-type and the new TTLL12 36AA plus isoform. Secondary structures of the (A) wild-type and (B) new 36AA plus isoform of TTLL12 protein were predicted by online tool CFSSP. The orange box indicates the inserted 36AA peptide.

A

TTLL12

B2L11 disordered region

B2L11 full amino acid length 1

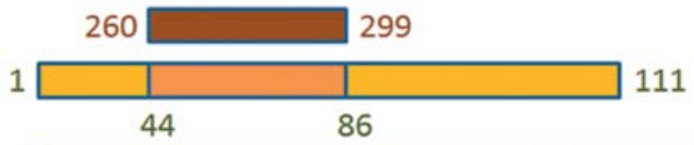

\B2L11

(134 aa)

Smith-Waterman score: 75 ;

Z-score: 116.9;

bits: 25.6 ;

$30.2 \%$ identity ( $53.5 \%$ similar) in 43 aa overlap (260-299: $44-86)$

TTLL12 260 APTDM_LDLSSCTPEPPAE HYQAI

:. : : : . : : : : . : : .

B2L11 12----REGRQLQPAERPPQLRPGAPTSLQTEPQDRSPAPMSCDKSTQTPSPPCQAFNHYLSA

TTLL12

LEE NK EKL PLD I NPVVH

B2L11

$$
: \text { : : : . }
$$

MASMRQAEPADMRPEIWIAQELRRIGDEFNAYYARRVFLNNYQAAEDHPRMV---121

\section{B}

The additional 36 amino acids BNP-32 disordered region

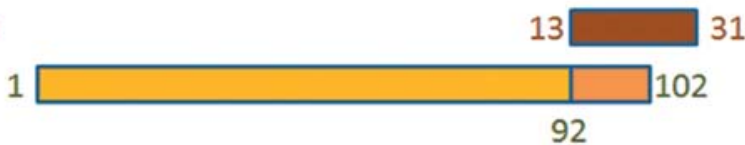

BNP-32 full amino acid length 1

>>BNP-32

(134 aa)

Smith-Waterman score: 47 ;

Z-score: 87.4 ;

bits: 20.0 ;

$42.1 \%$ identity (63.2\% similar) in 19 aa overlap (13-31: 92-110)

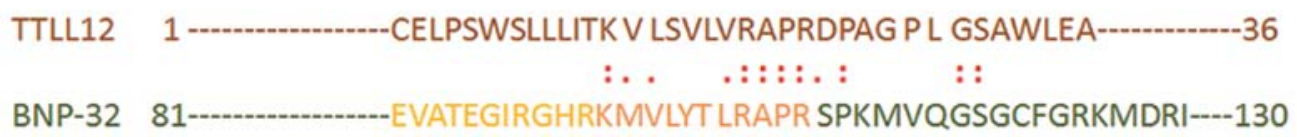

Figure 7. Analysis of the disordered region of the new TTLL12 36AA plus isoform. (A) The disordered region of the TTLL12 36AA plus isoform was predicted by entering the total TTLL12 amino acid sequence length into the opening accessible online resource DisProt search. The region from amino acids 260-299 of TTLL12 was highly aligned with the disordered region of Bcl-2-like protein 11 (B2L11). (B) The additional 36AA in the TTLL12 36AA plus isoform was pasted into DisProt search and showed that it was partly aligned with the disordered region of brain natriuretic peptide-32 (BNP-32).

TTLL12 36AA plus isoform. As the additional 36AA are located between 281AA and 282AA in the TTLL12 36AA plus isoform, we further explored whether the additional 36AA was in the disordered region of the protein. Using DisProt search, we found 
Table I. Physicochemical properties of the wild-type and new 36AA plus isoform of TTLL12.

\begin{tabular}{lcr}
\hline Physicochemical properties & Wild-type & New 36AA plus isoform \\
\hline Number of amino acids & 644 & 680 \\
Molecule formulas & $\mathrm{C}_{3359} \mathrm{H}_{5101} \mathrm{~N}_{905} \mathrm{O}_{959} \mathrm{~S}_{28}$ & $\mathrm{C}_{3537} \mathrm{H}_{5388} \mathrm{~N}_{950} \mathrm{O}_{1006} \mathrm{~S}_{29}$ \\
Molecular weight & $74,403.6$ & $78,245.1$ \\
PI & 5.33 & 5.34 \\
Instability index & 50.17 & 49.77 \\
Aliphatic index & 79.18 & 82.01 \\
Negatively charged residues (Asp + Glu) & 93 & 96 \\
Positively charged residues (Arg + Lys) & 65 & 68 \\
GRAVY & -0.390 & -0.338
\end{tabular}

TTLL, tubulin tyrosine ligase; PI, isoelectric point; GRAVY, grand average of hydropathicity.

Table II. Secondary structures of the TTLL12 wild-type and new 36AA plus isoform.

\begin{tabular}{|c|c|c|c|c|c|c|}
\hline \multirow[b]{2}{*}{ TTLL12 type } & \multicolumn{2}{|c|}{$\alpha$-Helix } & \multicolumn{2}{|c|}{$\beta$-sheet } & \multicolumn{2}{|c|}{$\beta$-turn } \\
\hline & Total residues & $\%$ & Total residues & $\%$ & Total residues & $\%$ \\
\hline Wild-type & 476 & 73.9 & 428 & 66.6 & 72 & 11.2 \\
\hline New 36AA plus isoform & 504 & 74.1 & 455 & 66.9 & 75 & 11.0 \\
\hline
\end{tabular}

TTLL, tubulin tyrosine ligase.

that the additional 36AA was partly identical to the disordered region of brain natriuretic peptide-32 (BNP-32) (Fig. 7B), giving the possibility that the 36AA could endow TTLL12 with new functions by acting as part of the disordered region. However, the biological functions of the new TTLL12 isoform in cancers remain to be further investigated.

\section{Discussion}

The tubulin tyrosine ligase (TTLL) family is a superfamily consisting of 14 members and they mainly catalyze ligation of amino acids to tubulins (1-3). TTLL12 is the least characterized and a most special member of the TTLL family, and it plays an important role in histone and tubulin modifications (14), mitotic duration and chromosome ploidy (5-8). It is the only member of the TTLL family without an assigned enzymatic function (15-17), and is said to be a pseudo-enzyme that has a phylogenetically conserved association of two domains including the SET-like domain and TTL-like domain in the $\mathrm{N}$ - and C-terminal of TTLL12, respectively. These two domains are related to histone methylation (18-22) and tubulin modifications (23-25) and then may contribute to tumorigenesis (4). The TTLL family is often suppressed in human cancers (10) and is closely connected with the poor prognosis of breast cancer (11), suggesting that TTLL12 could also play roles in human cancers. It is reported that TTLL12 increases its expression in the proliferating layer of benign human prostate and more apparently during cancer progression to metastasis (12), suggesting that TTLL12 may be of importance in tumor progression.
In the present study, we found an aberrant TTLL12 transcript with an additional 108-bp nucleotide sequence inserted between exons 5 and 6 . This new isoform, named the TTLL12 36AA plus isoform, exhibited much more abundance, as compared with the TTLL12 wild-type, in human cancer cells as to normal cells, particularly in human lung cancer cells, suggesting it could be be considered a new mechanism of human cancer development.

Alternatively splicing, by removing non-coding sequences (introns) and joining a coding part (exons) (26), plays an important role in gene expression (27-29). By the alternative splicing of precursor mRNAs (pre-mRNAs), multiple mRNAs and proteins can be generated from a single gene, and then the coding capacity of genomes is expanded through such a major mechanism (27-31). Furthermore, it has been reported that aberrant alternative splicing underlies various pathological processes, in particular tumorigenesis and tumor progression (32-34). The new TTLL12 36AA plus isoform may be the result of aberrant alternative splicing. Analysis by bioinformatics software showed that there are some differences in peptide physicochemical properties and protein secondary structure between the TTLL12 36AA plus isoform and the TTLL12 wild-type, suggesting that the new 36AA plus isoform could add some distinguished functions to TTLL12.

The traditional pattern analysis of protein structural biology indicates that the well-defined three-dimensional structures are pivotal to biological functions of proteins. However, it has been reported that the disordered regions of proteins are challenging this traditional structure-function paradigm $(35,36)$. Disordered regions are ubiquitous in cellular processes and 
human pathological conditions, but lack a well-defined, stable three-dimensional fold $(37,38)$. Proteins with disordered regions often go through disorder-to-order when binding to their partners and they can remain partially or fully flexible in their bound state, and form fuzzy complexes (37,39-41). In contrast, proteins with disordered regions are mainly involved in enzymatic activity $(37,42)$, including cell cycle regulation (43), cell division and differentiation $(42,44)$, which play critical roles in different types of cancers (45). In the present study, we report that the additional 36AA in the newly found TTLL12 protein isoform is a part of the disordered region, with the identity of those disordered regions identified in other functional proteins. Whether the 36AA is the binding site for certain proteins, such as those participating in posttranslational modification of tubulin or other important cell process, still needs to be investigated.

The TTLL family has been shown to be partially co-localized with vimentin and tubulin, which causes post-translational modifications of tubulin (5-8). Tubulin is a well acknowledged and important target for tumor therapy (46), but the precise functions of TTLL12 in tubulin modification are still poorly understood. Proteins related to post-translational modification are targets for new cancer therapeutic agents $(47,48)$, thus the new 36AA plus isoform of TTLL12 protein may have the potential to become a novel strategy for cancer treatment involving tubulin post-translational modifications. Therefore, the functions and molecular mechanisms of the new 36AA plus isoform in cancer development remain to be investigated.

In conclusion, our findings unveil a novel transcript isoform of TTLL12 with an additional 108-bp nucleotide sequence in the CDS. This TTLL12 36AA plus isoform could shed light on a novel mechanism of TTLL12 in human carcinogenesis and tumor progression, which may lead to a breakthrough of new potential targets for human cancer therapeutics.

\section{Acknowledgements}

The present study was supported by grants from the Science and Information Technology Bureau of Guangzhou (2011Y1-00022 and 2012-224-8, to J.T.), the Science and Information Technology Bureau of Guangzhou Haizhu District (2012-ZD-02, to J.T.), and the Guangzhou Medical University (B147048, to J.T.).

\section{References}

1. Janke C, Rogowski K, Wloga D, Regnard C, Kajava AV, Strub JM, Temurak N, van Dijk J, Boucher D, van Dorsselaer A, et al: Tubulin polyglutamylase enzymes are members of the TTL domain protein family. Science 308: 1758-1762, 2005.

2. Fukushima N, Furuta D, Hidaka Y, Moriyama R and Tsujiuchi T: Post-translational modifications of tubulin in the nervous system. J Neurochem 109: 683-693, 2009.

3. Ersfeld K, Wehland J, Plessmann U, Dodemont H, Gerke V and Weber K: Characterization of the tubulin-tyrosine ligase. J Cell Biol 120: 725-732, 1993.

4. Brants J, Semenchenko K, Wasylyk C, Robert A, Carles A, Zambrano A, Pradeau-Aubreton K, Birck C, Schalken JA, Poch O, et al: Tubulin tyrosine ligase like 12, a TTLL family member with SET- and TTL-like domains and roles in histone and tubulin modifications and mitosis. PLoS One 7: e51258, 2012.

5. Gurland G and Gundersen GG: Stable, detyrosinated microtubules function to localize vimentin intermediate filaments in fibroblasts. J Cell Biol 131: 1275-1290, 1995.
6. Peris L, Thery M, Fauré J, Saoudi Y, Lafanechère L, Chilton JK, Gordon-Weeks P, Galjart N, Bornens M, Wordeman L, et al: Tubulin tyrosination is a major factor affecting the recruitment of CAP-Gly proteins at microtubule plus ends. J Cell Biol 174: 839-849, 2006.

7. Dunn S, Morrison EE, Liverpool TB, Molina-París C, Cross RA, Alonso MC and Peckham M: Differential trafficking of Kif5c on tyrosinated and detyrosinated microtubules in live cells. J Cell Sci 121: 1085-1095, 2008.

8. Gyoeva FK and Gelfand VI: Coalignment of vimentin intermediate filaments with microtubules depends on kinesin. Nature 353: 445-448, 1991.

9. Kato C, Miyazaki K, Nakagawa A, Ohira M, Nakamura Y, Ozaki T, Imai T and Nakagawara A: Low expression of human tubulin tyrosine ligase and suppressed tubulin tyrosination/detyrosination cycle are associated with impaired neuronal differentiation in neuroblastomas with poor prognosis. Int $\mathbf{J}$ Cancer 112: 365-375, 2004.

10. Lafanechère L, Courtay-Cahen C, Kawakami T, Jacrot M, Rüdiger M, Wehland J, Job D and Margolis RL: Suppression of tubulin tyrosine ligase during tumor growth. J Cell Sci 111: 171-181, 1998.

11. Mialhe A, Lafanechère L, Treilleux I, Peloux N, Dumontet C, Brémond A, Panh MH, Payan R, Wehland J, Margolis RL, et al: Tubulin detyrosination is a frequent occurrence in breast cancers of poor prognosis. Cancer Res 61: 5024-5027, 2001.

12. Wasylyk C, Zambrano A, Zhao C, Brants J, Abecassis J, Schalken JA, Rogatsch H, Schaefer G, Pycha A, Klocker H, et al: Tubulin tyrosine ligase like 12 links to prostate cancer through tubulin posttranslational modification and chromosome ploidy. Int J Cancer 127: 2542-2553, 2010.

13. Soucek K, Kamaid A, Phung AD, Kubala L, Bulinski JC, Harper RW and Eiserich JP: Normal and prostate cancer cells display distinct molecular profiles of $\alpha$-tubulin posttranslational modifications. Prostate 66: 954-965, 2006.

14. Szyk A, Deaconescu AM,Piszczek G and Roll-Mecak A: Tubulin tyrosine ligase structure reveals adaptation of an ancient fold to bind and modify tubulin. Nat Struct Mol Biol 18: 1250-1258, 2011.

15. Ikegami $\mathrm{K}$ and Setou M: TTLL10 can perform tubulin glycylation when co-expressed with TTLL8. FEBS Lett 583: 1957-1963, 2009.

16. Wloga D, Webster DM, Rogowski K, Bré MH, Levilliers N Jerka-Dziadosz M, Janke C, Dougan ST and Gaertig J: TTLL3 is a tubulin glycine ligase that regulates the assembly of cilia. Dev Cell 16: 867-876, 2009.

17. Rogowski K, Juge F, van Dijk J, Wloga D, Strub JM, Levilliers N, Thomas D, Bré MH, Van Dorsselaer A, Gaertig J, et al: Evolutionary divergence of enzymatic mechanisms for posttranslational polyglycylation. Cell 137: 1076-1087, 2009.

18. Dillon SC, Zhang X, Trievel RC and Cheng X: The SET-domain protein superfamily: Protein lysine methyltransferases. Genome Biol 6: 227, 2005.

19. Cheng $X$ and Zhang X: Structural dynamics of protein lysine methylation and demethylation. Mutat Res 618: 102-115, 2007.

20. Xu D, Bai J, Duan Q, Costa M and Dai W: Covalent modifications of histones during mitosis and meiosis. Cell Cycle 8: 3688-3694, 2009.

21. Qian C and Zhou MM: SET domain protein lysine methyltransferases: Structure, specificity and catalysis. Cell Mol Life Sci 63: 2755-2763, 2006.

22. Aravind L, Abhiman S and Iyer LM: Natural history of the eukaryotic chromatin protein methylation system. Prog Mol Biol Transl Sci 101: 105-176, 2011.

23. Garnham CP and Roll-Mecak A: The chemical complexity of cellular microtubules: Tubulin post-translational modification enzymes and their roles in tuning microtubule functions. Cytoskeleton Hoboken 69: 442-463, 2012.

24. Wloga D and Gaertig J: Post-translational modifications of microtubules. J Cell Sci 123: 3447-3455, 2010.

25. Janke $C$ and Bulinski JC: Post-translational regulation of the microtubule cytoskeleton: Mechanisms and functions. Nat Rev Mol Cell Biol 12: 773-786, 2011.

26. Sharp PA: Split genes and RNA splicing. Cell 77: 805-815, 1994.

27. Yang X, Coulombe-Huntington J, Kang S, Sheynkman GM, Hao T, Richardson A, Sun S, Yang F, Shen YA, Murray RR, et al: Widespread expansion of protein interaction capabilities by alternative splicing. Cell 164: 805-817, 2016. 
28. Du Q, Li C, Li D and Lu S: Genome-wide analysis, molecular cloning and expression profiling reveal tissue-specifically expressed, feedback-regulated, stress-responsive and alternatively spliced novel genes involved in gibberellin metabolism in Salvia miltiorrhiza. BMC Genomics 16: 1087, 2015.

29. Rodríguez SA, Grochová D, McKenna T, Borate B, Trivedi NS, Erdos MR and Eriksson M: Global genome splicing analysis reveals an increased number of alternatively spliced genes with aging. Aging Cell 15: 267-278, 2016.

30. Shang J, Fan X, Shangguan L, Liu H and Zhou Y: Global gene expression profiling and alternative splicing events during the chondrogenic differentiation of human cartilage endplate-derived stem cells. BioMed Res Int 2015: 604972, 2015.

31. Stevens M and Oltean S: Alternative splicing in CKD. J Am Soc Nephrol 27: 1596-1603, 2016.

32. Sheng Z, Sun Y, Zhu R, Jiao N, Tang K, Cao Z and Ma C: Functional cross-talking between differentially expressed and alternatively spliced genes in human liver cancer cells treated with berberine. PLoS One 10: e0143742, 2015.

33. Adamopoulos PG, Kontos CK, Tsiakanikas P and Scorilas A: Identification of novel alternative splice variants of the $B C L 2 L 12$ gene in human cancer cells using next-generation sequencing methodology. Cancer Lett 373: 119-129, 2016.

34. Faustino NA and Cooper TA: Pre-mRNA splicing and human disease. Genes Dev 17: 419-437, 2003

35. Forman-Kay JD and Mittag T: From sequence and forces to structure, function, and evolution of intrinsically disordered proteins. Structure 21: 1492-1499, 2013.

36. Dunker AK, Bondos SE, Huang F and Oldfield CJ: Intrinsically disordered proteins and multicellular organisms. Semin Cell Dev Biol 37: 44-55, 2015.

37. Wright PE and Dyson HJ: Intrinsically unstructured proteins: Re-assessing the protein structure-function paradigm. J Mol Biol 293: 321-331, 1999.
38. Chouard T: Structural biology: Breaking the protein rules. Nature 471: 151-153, 2011.

39. Kotta-Loizou I, Tsaousis GN and Hamodrakas SJ: Analysis of molecular recognition features (MoRFs) in membrane proteins. Biochim Biophys Acta 1834: 798-807, 2013.

40. Tompa P and Fuxreiter M: Fuzzy complexes: Polymorphism and structural disorder in protein-protein interactions. Trends Biochem Sci 33: 2-8, 2008.

41. Fuxreiter M and Tompa P: Fuzzy complexes: A more stochastic view of protein function. Adv Exp Med Biol 725: 1-14, 2012.

42. Xie H, Vucetic S, Iakoucheva LM, Oldfield CJ, Dunker AK, Uversky VN and Obradovic Z: Functional anthology of intrinsic disorder. 1. Biological processes and functions of proteins with long disordered regions. J Proteome Res 6: 1882-1898, 2007.

43. Yoon MK, Mitrea DM, Ou L and Kriwacki RW: Cell cycle regulation by the intrinsically disordered proteins p21 and p27. Biochem Soc Trans 40: 981-988, 2012.

44. Ward JJ, Sodhi JS, McGuffin LJ, Buxton BF and Jones DT: Prediction and functional analysis of native disorder in proteins from the three kingdoms of life. J Mol Biol 337: 635-645, 2004.

45. Andresen C, Helander S, Lemak A, Farès C, Csizmok V, Carlsson J, Penn LZ, Forman-Kay JD, Arrowsmith CH, Lundström $\mathrm{P}$, et al: Transient structure and dynamics in the disordered c-Myc transactivation domain affect Bin1 binding. Nucleic Acids Res 40: 6353-6366, 2012.

46. Harrison MR, Holen KD and Liu G: Beyond taxanes: A review of novel agents that target mitotic tubulin and microtubules, kinases, and kinesins. Clin Adv Hematol Oncol 7: 54-64, 2009.

47. Verhey KJ and Gaertig J: The tubulin code. Cell Cycle 6: 2152-2160, 2007

48. Ghoreschi K, Laurence A and O'Shea JJ: Selectivity and therapeutic inhibition of kinases: To be or not to be? Nat Immunol 10: 356-360, 2009. 